\title{
Magnetic Density of States at Low Energy in Geometrically Frustrated Systems
}

\author{
A. Yaouanc, ${ }^{1}$ P. Dalmas de Réotier, ${ }^{1}$ V. Glazkov, ${ }^{1}$ C. Marin, ${ }^{1}$ P. Bonville, ${ }^{2}$ J. A. Hodges, ${ }^{2}$ P. C. M. Gubbens, ${ }^{3}$ \\ S. Sakarya, ${ }^{3}$ and C. Baines ${ }^{4}$ \\ ${ }^{1}$ CEA/DSM/Département de Recherche Fondamentale sur la Matière Condensée, 38054 Grenoble, France \\ ${ }^{2}$ CEA/DSM/Département de Recherche sur l'Etat Condensée, les Atomes et les Molécules, 91191 Gif sur Yvette, France \\ ${ }^{3}$ Department of Radiation, Radionuclides \& Reactors, Delft University of Technology, $2629 \mathrm{JB}$ Delft, The Netherlands \\ ${ }^{4}$ Laboratory for Muon-Spin Spectroscopy, Paul Scherrer Institute, 5232 Villigen-PSI, Switzerland
} (Received 4 February 2005; published 22 July 2005)

\begin{abstract}
Using muon-spin-relaxation measurements we show that the pyrochlore compound $\mathrm{Gd}_{2} \mathrm{Ti}_{2} \mathrm{O}_{7}$, in its magnetically ordered phase below $\sim 1 \mathrm{~K}$, displays persistent spin dynamics down to temperatures as low as $20 \mathrm{mK}$. The characteristics of the induced muon relaxation can be accounted for by a scattering process involving two magnetic excitations, with a density of states characterized by an upturn at low energy and a small gap depending linearly on the temperature. We propose that such a density of states is a generic feature of geometrically frustrated magnetic materials.
\end{abstract}

The study of geometrically frustrated materials (GFMs) is a subject at the forefront of research in condensed matter physics not only because of their own interest but also because the concept of the frustration of the interactions plays a role for understanding the physics of, e.g., ice, cholesteric crystals, and metallic glasses; see, for instance, Ref. [1] for a discussion.

Magnetic materials based on lattices with triangular motifs and nearest-neighbor antiferromagnetic exchange interaction belong to the family of GFMs. They are, in the absence of further terms in the expression of their energy, believed to remain disordered and fluctuating down to zero temperature [2]. The absence of magnetic order stems from their highly degenerate ground state. It is only upon inclusion of perturbations such as exchange interactions extending beyond nearest-neighbor magnetic ions or dipole coupling that magnetic ordering may appear. Experiments on Kagomé, garnet, and pyrochlore structure compounds support these general predictions [1].

A fingerprint for the geometrical frustration of a material is the shift towards low energy of the spectral weight of excitations [1]. The first convincing experimental proof has been obtained in a Kagomé-like magnetic material, where the low temperature specific heat [3] is found to be dominated by singlet excitations arising from correlated spins, rather than from individual spins. This enhanced density of spectral weight at low energy could be linked with the persistence of spin dynamics observed at low temperature in many systems, as magnetic excitations are continuously available from zero energy. This behavior has been evidenced in the Kagomé compounds $\mathrm{SrCr}_{9 p} \mathrm{Ga}_{12-9 p} \mathrm{O}_{19}$ with $0.39 \leq p \leq 0.89$ [4,5], for which a spin-glass transition is detected at $T_{\mathrm{g}}=4 p \mathrm{~K}$, in Kagomé-like systems [6,7], in the garnet $\mathrm{Gd}_{3} \mathrm{Ga}_{5} \mathrm{O}_{12}$ [8-10] with $T_{\mathrm{g}}=0.15 \mathrm{~K}$, and in the pyrochlore compounds $\mathrm{Y}_{2} \mathrm{Mo}_{2} \mathrm{O}_{7} \quad\left(T_{\mathrm{g}}=22 \mathrm{~K}\right)$ [11], $\mathrm{Tb}_{2} \mathrm{Mo}_{2} \mathrm{O}_{7}\left(T_{\mathrm{g}}=25 \mathrm{~K}\right)$ [11]. It was also observed in the spin liquid $\mathrm{Tb}_{2} \mathrm{Ti}_{2} \mathrm{O}_{7}$ [12-14], for which there is no evi- dence of a transition down to $0.07 \mathrm{~K}$ [15]. The spin dynamics becomes approximately temperature independent below about $T_{\mathrm{g}}$ for the spin-glass systems, except for the two molybdates for which it occurs in the temperature range $T / T_{\mathrm{g}}<0.05$, and for $\mathrm{Tb}_{2} \mathrm{Ti}_{2} \mathrm{O}_{7}$ which displays a temperature independent relaxation below about $1 \mathrm{~K}$. Appreciable spin dynamics is also found in $\mathrm{Yb}_{2} \mathrm{Ti}_{2} \mathrm{O}_{7}$ below the temperature at which the specific heat presents a sharp anomaly [16-18]. In compounds where the fluctuations have been studied by the muon-spin-relaxation ( $\mu \mathrm{SR})$ technique, the muon-spin-lattice relaxation function is usually found to be a stretched exponential, i.e., $P_{Z}^{\mathrm{sl}}(t)=\exp \left[-\left(\lambda_{Z} t\right)^{\alpha}\right]$ with $\alpha \neq 1$.

More unconventional is the spin dynamics recently observed by Mössbauer spectroscopy, down to $30 \mathrm{mK}$, in $\mathrm{Gd}_{2} \mathrm{Sn}_{2} \mathrm{O}_{7}$ which shows long-range ordering below $1 \mathrm{~K}$ [19], and which was confirmed by $\mu \mathrm{SR}$ measurements down to $20 \mathrm{mK}[20,21]$. This anomalous spin dynamics cannot arise from conventional magnons, since their population vanishes at low temperature. In order to further investigate these zero temperature fluctuations, we performed $\mu \mathrm{SR}$ measurements in the parent compound $\mathrm{Gd}_{2} \mathrm{Ti}_{2} \mathrm{O}_{7}$, for which single crystals are available. This compound undergoes a first magnetic transition at $T_{\mathrm{cl}} \simeq$ $1 \mathrm{~K}$, followed by a second one at $T_{\mathrm{c} 2} \simeq 0.75 \mathrm{~K}$ [22]. We report the results of specific heat and $\mu \mathrm{SR}$ measurements in this material, and propose to account for the observed persistent spin dynamics in GFMs in terms of an unconventional density of states at low energy.

A $\mathrm{Gd}_{2} \mathrm{Ti}_{2} \mathrm{O}_{7}$ polycrystalline rod was prepared by mixing, heating, and compacting the constituent oxides $\mathrm{Gd}_{2} \mathrm{O}_{3}$ and $\mathrm{TiO}_{2}$ of respective purity $5 \mathrm{~N}$ and $4 \mathrm{~N} 5$. A single crystal was then grown by the traveling solvent floating zone technique using a Crystal System Inc. optical furnace with a velocity of $8 \mathrm{~mm}$ per hour. Oriented platelets were cut from the crystal and subsequently annealed under oxygen pressure to ensure optimized physical properties. The 
top panel of Fig. 1 shows the result of specific heat measurements performed using a dynamic adiabatic technique. The two phase transitions are observed at $T_{\mathrm{c} 1}=1.02 \mathrm{~K}$ and $T_{\mathrm{c} 2}=0.74 \mathrm{~K}$, respectively, in agreement with recent single crystal measurements [23]. The peak at $T_{\mathrm{c} 2}$ is found much sharper than previously reported [22-24]. Its shape suggests that the lower phase transition is first order. Although we do not have any experimental evidence, it is possible that at this lower magnetic transition corresponds a structural transition which could be induced through magneto-elastic coupling. Anyhow, the magnetic transitions do not relieve the frustration since in the following we do report the observation of persistent spin dynamics, a fingerprint of geometrical magnetic frustration. At low temperature, up to $\sim 0.55 \mathrm{~K}$, the specific heat divided by temperature $C_{\mathrm{p}} / T$ is proportional to $T$. Such a behavior was also found for $\mathrm{Gd}_{2} \mathrm{Sn}_{2} \mathrm{O}_{7}$ [24] and Kagomé-like compounds [3], but it is not a general rule for GFMs since a $T^{2}$ dependence is reported for $\mathrm{Er}_{2} \mathrm{Ti}_{2} \mathrm{O}_{7}$ [25]. The entropy release reaches $\simeq 90 \%$ of $R \ln 8$ only near $5 \mathrm{~K}$.

The $\mu$ SR measurements (see Ref. [26] for an introduction to this technique) were performed at the Low Temperature Facility ( $\pi \mathrm{M} 3$ beam line) of the Swiss Muon Source (Paul Scherrer Institute, Villigen, Switzerland). As expected, the $\mu$ SR spectra in the paramagnetic phase are well described by a single exponential relaxation function; i.e., they are proportional to $P_{Z}^{\exp }(t)=$ $\exp \left(-\lambda_{Z} t\right)$, where $\lambda_{Z}$ is the spin-lattice relaxation rate. $Z$

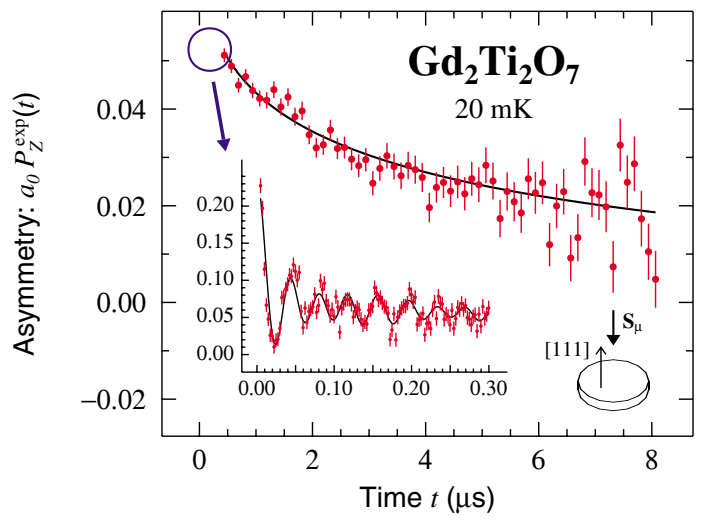

FIG. 1 (color online). $\mu \mathrm{SR}$ spectrum recorded in zero field at $20 \mathrm{mK}$ in a single crystal of $\mathrm{Gd}_{2} \mathrm{Ti}_{2} \mathrm{O}_{7}$ with the initial muon beam polarization parallel to the [111] crystal direction. The main frame displays the time spectrum up to $8 \mu \mathrm{s}$, and the inset magnifies the short time behavior. The latter is characterized by wiggles which are damped out at the earliest time displayed in the main frame. The wiggles correspond to the spontaneous precession of the muon spin in two local fields of respective magnitude $B_{1}=194(1)$ and $B_{2}=149$ (1) $\mathrm{mT}$. As expected, the spin-lattice relaxation channel, most easily seen in the main frame, accounts for $\sim 1 / 3$ of the spectrum and the wiggles for $\sim 2 / 3$, this being temperature independent as is also the total asymmetry. The amplitude of the $B_{1}$ component is approximately 4 times as large as that of $B_{2}$. labels the direction of the initial muon beam polarization. Below $T_{\mathrm{c} 1}$, the observed oscillations, due to spontaneous precession of the muon spin (see inset of Fig. 2), are a signature of the long-range order of the magnetic structure, consistent with the observation of magnetic Bragg reflections by neutron diffraction [27,28]. Surprisingly, even at $20 \mathrm{mK}$, a stretched exponential decay of the spin-lattice relaxation channel is observed, superposed on the damped wiggles (Fig. 2). This implies that excitations of the spin



FIG. 2 (color online). In $\mathrm{Gd}_{2} \mathrm{Ti}_{2} \mathrm{O}_{7}$, low temperature dependence of specific heat over temperature $C_{p} / T$ per mole of $\mathrm{Gd}$ (top panel), of the muon-spin-lattice relaxation rate $\lambda_{Z}$ (middle panel), and of the exponent $\alpha$ (bottom panel) of the stretched exponential decay. The muon data were measured in a single crystal, with different orientations relative to the muon polarization $\mathbf{S}_{\mu}$, as shown, and the anisotropy of $\lambda_{Z}$ was found to be negligible. The dashed lines for $\lambda_{Z}(T)$ and $\alpha(T)$ are guides for the eye. The lower inset shows, on a double logarithmic scale, the weak temperature dependence of $\lambda_{Z}(T)$ at low temperature. The full lines give the predictions for $C_{p} / T$ and $\lambda_{Z}$ computed with $b_{\mu}=0.03 \mathrm{meV}^{-1 / 2}, b_{\mathrm{sh}}=26 \mathrm{meV}^{-2}$ per $\mathrm{Gd}$ atom and $a=0.1$ (see text). The upper inset shows the density of magnetic excitations $g_{m}(\epsilon)$ at $50 \mathrm{mK}$, with its two components as dashed lines. The gap is: $\Delta \simeq 5 \times 10^{-4} \mathrm{meV}$. The upturn at low energy is responsible for the finite value of $\lambda_{Z}$ as $T \rightarrow 0$. 
system are present, inducing spin-lattice relaxation of the muon levels. In Fig. 1 are displayed the thermal variations of the two parameters accounting for this channel, $\lambda_{Z}$, and the stretched exponent $\alpha$.

Approaching $T_{\mathrm{c} 1}$ from above, $\lambda_{Z}$ increases, reflecting the slowing down of the paramagnetic fluctuations. As expected, it drops when crossing $T_{\mathrm{cl}}$, but it remains roughly temperature independent below $0.5 \mathrm{~K}$ with a value $\lesssim 1 \mu \mathrm{s}^{-1}$. So the persistent spin dynamics known to exist in GFMs with no long-range magnetic order is also present in a magnetically ordered compound. Interestingly, $\alpha$ is not far from $1 / 2$ for $T<T_{\mathrm{c} 2}$ and jumps to $\sim 3 / 4$ for $T_{\mathrm{c} 2}<T<$ $T_{\mathrm{c} 1}$. It is expected to be equal to 1 for a homogeneous spin system, as found in the paramagnetic state. A value $\alpha=$ $1 / 2$ suggests that relaxation stems from only a small fraction $c$ of the spins [29], fluctuating with a correlation time $\tau_{c}$ and distributed at random in the lattice. These spins create a field distribution at the muon site which is known to have a squared Lorentzian shape [30], with a width $\Delta_{\text {Lor }}$. A recent neutron diffraction study of $\mathrm{Gd}_{2} \mathrm{Ti}_{2} \mathrm{O}_{7}$ [28] has shown that $1 / 4$ of the $\mathrm{Gd}$ magnetic moments are only partially ordered in the low temperature phase, and we tentatively attribute the relaxation channel with $\alpha=1 / 2$ to a fraction of these spins. Our measurements at $0.1 \mathrm{~K}$ with a longitudinal field (not shown) yield $\tau_{c}=0.7(2) \mathrm{ns}$ and the relationship $\lambda_{Z}=4 \Delta_{\text {Lor }}^{2} \tau_{c}$ yields a width $\Delta_{\text {Lor }}=$ 18(5) mT. According to Uemura et al. [31], $\Delta_{\text {Lor }}=$ $\sqrt{\pi / 2} c \Delta_{\max }$, where $\Delta_{\max }$ is the field width if all the Gd moments were contributing to the muon relaxation. Using the scaling between $\Delta_{\max }$ and the rare earth moment which was successfully used for $\mathrm{Tb}_{2} \mathrm{Ti}_{2} \mathrm{O}_{7}$ [12] and $\mathrm{Yb}_{2} \mathrm{Ti}_{2} \mathrm{O}_{7}$ [17], we deduce $c \leqslant 10 \%$. We note that a significant distribution in the spin fluctuation times has been observed, by the neutron spin echo technique, in $\mathrm{Gd}_{2} \mathrm{Ti}_{2} \mathrm{O}_{7}$ [32] and in $\mathrm{Tb}_{2} \mathrm{Ti}_{2} \mathrm{O}_{7}$ [13]. This backs our observation that only a fraction of the $\mathrm{Gd}^{3+}$ ions relax the muon spin, whereas the remaining part does not because it is characterized by fluctuation times either too short or too long.

Our specific heat and $\mu$ SR measurements show therefore that, in the long-range order phase of $\mathrm{Gd}_{2} \mathrm{Ti}_{2} \mathrm{O}_{7}$, magnetic excitations with a nonvanishing density at low energy are present. These excitations lead to a $T^{2}$ behavior for the specific heat and to a muon spin-lattice relaxation rate which is quasi-independent of temperature. In fact, below $0.5 \mathrm{~K}$ a fit of $\lambda_{Z}(T)$ to a power law gives $\lambda_{Z}(T) \propto T^{\beta}$ with $\beta \simeq 1 / 3$. This is a negligible temperature dependence relative to power laws observed for usual ferromagnets $(\beta=2)$ and conventional antiferromagnets $(\beta=5)$ [21]. In the following, we aim at determining the density of magnetic excitations responsible for the observed thermal behaviors of $C_{p}$ and $\lambda_{Z}$.

First of all, assuming these excitations to obey BoseEinstein statistics like conventional magnons, it is easy to derive that, if their density of states per volume unit $g_{m}(\epsilon)$ is proportional to $\epsilon^{q}$, then $C_{p} \propto T^{q+1}$. Hence $g_{m}(\epsilon) \propto \epsilon$ accounts for the $T^{2}$ behavior of $C_{p}$. Second, for the muon relaxation rate, if one considers a direct process with a single excitation, then energy conservation with $\epsilon=\epsilon_{\mu} \simeq 0$ ( $\epsilon_{\mu}=\hbar \omega_{\mu}, \omega_{\mu}$ being the muon angular frequency) leads to $\lambda_{Z} \propto T$, which is in disagreement with the experimental data for $\mathrm{Gd}_{2} \mathrm{Ti}_{2} \mathrm{O}_{7}$ and other frustrated systems mentioned in the introduction. The relaxation process to consider next involves a two-excitation scattering (Raman process). Within the harmonic approximation, energy conservation implies that only the component of the spin-spin correlation tensor parallel to the magnetization, $\Lambda^{\|}\left(\mathbf{q}, \omega_{\mu}=0\right)$, is probed [33]. Thus, $\lambda_{Z} \propto \int C(\mathbf{q}) \Lambda^{\|}\left(\mathbf{q}, \omega_{\mu}=0\right) \mathrm{d}^{3} \mathbf{q}$, where $C(\mathbf{q})$ accounts for the interaction of the muon spin with the lattice spins and the integral extends over the Brillouin zone. For antiferromagnets with two collinear sublattices, the muon relaxation rate due to a Raman magnon process has been derived assuming for simplicity no orientation dependence for $\epsilon(q)$ and $C(q)$ :

$$
\begin{aligned}
\lambda_{Z}= & \frac{8(2 \pi)^{3} \mathcal{D} \hbar}{15} \frac{\left(B_{e}+B_{a}\right)^{2}}{\left(B_{e}+B_{a}\right)^{2}-B_{e}^{2}} \int_{\Delta}^{\infty} n\left(\epsilon / k_{\mathrm{B}} T\right) \\
& \times\left[n\left(\epsilon / k_{\mathrm{B}} T\right)+1\right] g_{m}^{2}(\epsilon) \mathrm{d} \epsilon,
\end{aligned}
$$

where $\mathcal{D}=\left(\mu_{0} / 4 \pi\right)^{2} \gamma_{\mu}^{2} g^{2} \mu_{B}^{2}, n\left(\epsilon / k_{\mathrm{B}} T\right)$ is the BoseEinstein occupation factor, and $\Delta$ the energy gap of the excitations at zero energy. $B_{e}$ and $B_{a}$ are, respectively, the exchange and anisotropy fields; a mean-field estimate for $B_{e}$ is $10 \mathrm{~T}$ and, with reference to another Gd compound [34], we take $B_{a}=0.2$ T. Equation (1) contains two population factors standing for the creation and annihilation of an excitation, a density of states being associated with each of them. The expression given by Eq. (1) assumes a muon site of high symmetry, e.g., the octahedral or tetrahedral interstitial sites of a face centered cubic lattice. For a muon site of lower symmetry, as expected in the case of the pyrochlores, Eq. (1) is essentially modified by a multiplicative factor $\eta$ which depends on the actual site and is in the range between 1 and $\sim 10$. We took $\eta=7$ in the calculation below.

As a first step, let us assume $\lambda_{Z}$ to be temperature independent. The density of states must be $g_{m}(\epsilon)=$ $b_{\mu} \epsilon^{-1 / 2}$ [35]. It must also be assumed that $\Delta=a k_{\mathrm{B}} T$; i.e., the gap at zero energy is proportional to temperature. If $a$ is of order 1 or lower, it can be shown that $\lambda_{Z} \propto b_{\mu}^{2} / a^{2}$ and that $g_{m}(\epsilon)$ is essentially probed for $\Delta \leq \epsilon \lesssim 3 \Delta$. Therefore, the $\epsilon^{-1 / 2}$ dependence for $g_{m}(\epsilon)$ holds only in a very restricted energy interval. As stated above, the $T^{2}$ dependence of $C_{p}$ implies that $g_{m}(\epsilon)$ is linear with energy, and this must prevail for $\epsilon \gtrsim 3 \Delta$. Combining the two regimes, we obtain: $g_{m}(\epsilon)=b_{\mu} \epsilon^{-1 / 2}+b_{\mathrm{sh}} \epsilon$. The numerical calculations show that $a \leq 0.1$ is compatible with the $C_{p} / T$ and $\lambda_{Z}$ data. The predictions of the model with $a=$ 0.1 are shown in Fig. 1 . As expected, the model predicts $\lambda_{Z}$ to be independent of $T$ at low temperature. Note that a 
further refinement of the model, namely, a slight change in the value of the exponent in the former term of $g_{m}(\epsilon)$ $(-0.5 \rightarrow-0.4)$, allows us to improve the fit of $\lambda_{Z}(T)$ at low $T$ for the specific case of $\mathrm{Gd}_{2} \mathrm{Ti}_{2} \mathrm{O}_{7}$. This change affects the details in the shape of $g_{m}(\epsilon)$, but not its main features: the upturn at small energy and the existence of a gap proportional to the temperature.

The calculated $C_{p} / T$ shown in Fig. 1 is $T$ linear as expected, but shows an upturn below about $40 \mathrm{mK}$, caused by the $\epsilon^{-1 / 2}$ dependence of the density of states. This signature could be searched for in very low temperature specific heat measurements performed on a sample enriched with $\mathrm{Gd}$ isotopes having zero nuclear moment, thus showing no nuclear Schottky anomaly. The computed curve for $\lambda_{Z}$ above $\sim 0.4 \mathrm{~K}$ overestimates the measured values. This could indicate that a fraction of the density of states, in the region where the dependence $g_{m}(\epsilon) \propto \epsilon$ prevails, arises from singletlike states [3] which do not contribute to the relaxation.

In summary, we have shown that, in GFMs, a nonvanishing muon-spin-lattice relaxation at low $T$, with a weak temperature dependence, if any, is the signature of a low energy upturn in the density of magnetic states. This density is characterized by a gap varying linearly with temperature, leading to an accumulation of states at low energy (see the upper inset of Fig. 1). This is a rare feature, which is also observed in BCS superconductors. We propose that the gap is due to the dipole interaction between magnetic moments, which breaks rotational invariance. However, its increase with $T$ is unexpected. It is usually temperature independent or, for superconductors, decreases as temperature is increased [36]. A linear thermal increase strongly suggests that the thermal energy exceeds the energy involved in the excitation scattering process. Indeed, an analytical classical calculation performed for a triangular planar model with nearest-neighbor antiferromagnetic interactions and dipole interactions predicts a gap proportional to $T$ with $a=0.2$ for one of the phases [37].

As pointed out at the beginning of this Letter, persistent and weakly temperature spin dynamics has been observed for a large number of GFM, included magnetically ordered powder samples of $\mathrm{Gd}_{2} \mathrm{Ti}_{2} \mathrm{O}_{7}$ [17], $\mathrm{Gd}_{2} \mathrm{Sn}_{2} \mathrm{O}_{7}$ [10,21], and $\mathrm{Er}_{2} \mathrm{Ti}_{2} \mathrm{O}_{7}$ [38]. A combined analysis of $C_{p}(T)$ and $\lambda_{Z}(T)$ is always possible, resulting in $g_{m}$ with a gap linear in temperature.

The possibility for a GFM to order magnetically at finite temperature, although it is predicted to remain disordered down to zero temperature, was discovered theoretically a long time ago [39], as "order from thermal disorder." The gap we infer here is a consequence of the same mechanism. It appears if rotational invariance is broken. This naturally occurs because of the presence of the dipole interaction.

We thank B. Canals, G. Jackeli, and S. V. Maleyev for enlightening discussions. Part of this work was performed at the $\mathrm{S} \mu \mathrm{S}$, Paul Scherrer Institute, Villigen, Switzerland.
[1] A. P. Ramirez, in Handbook of Magnetic Materials, edited by K. H. J. Buschow (Elsevier, New York, 2001), Vol. 13.

[2] J. Villain, Z. Phys. B 33, 31 (1979).

[3] A. P. Ramirez, B. Hessen, and M. Winklemann, Phys. Rev. Lett. 84, 2957 (2000).

[4] Y. J. Uemura et al., Phys. Rev. Lett. 73, 3306 (1994).

[5] A. Keren et al., Phys. Rev. Lett. 84, 3450 (2000).

[6] A. Fukaya et al., Phys. Rev. Lett. 91, 207603 (2003).

[7] D. Bono et al., Phys. Rev. Lett. 93, 187201 (2004).

[8] S. R. Dunsiger et al., Phys. Rev. Lett. 85, 3504 (2000).

[9] I. M. Marshall et al., J. Phys. Condens. Matter 14, L157 (2002).

[10] P. Bonville et al., Phys. Rev. Lett. 92, 167202 (2004).

[11] S. R. Dunsiger et al., Phys. Rev. B 54, 9019 (1996).

[12] J. S. Gardner et al., Phys. Rev. Lett. 82, 1012 (1999).

[13] J. S. Gardner et al., Phys. Rev. B 68, 180401(R) (2003).

[14] A. Keren et al., Phys. Rev. Lett. 92, 107204 (2004).

[15] J. S. Gardner et al., Phys. Rev. B 64, 224416 (2001).

[16] J. Hodges et al., Phys. Rev. Lett. 88, 077204 (2002).

[17] A. Yaouanc et al., Physica B (Amsterdam) 326, 456 (2003).

[18] J. S. Gardner et al., Phys. Rev. B 70, 180404(R) (2004).

[19] E. Bertin et al., Eur. Phys. J. B 27, 347 (2002).

[20] P. Bonville et al., Hyperfine Interact. 156-157, 103 (2004).

[21] P. Dalmas de Réotier, P. C. M. Gubbens, and A. Yaouanc, J. Phys. Condens. Matter 16, S4687 (2004).

[22] A. P. Ramirez et al., Phys. Rev. Lett. 89, 067202 (2002).

[23] O. A. Petrenko et al., Phys. Rev. B 70, 012402 (2004).

[24] P. Bonville et al., J. Phys. Condens. Matter 15, 7777 (2003).

[25] J. D. M. Champion et al., Phys. Rev. B 68, 020401 (2003).

[26] P. Dalmas de Réotier and A. Yaouanc, J. Phys. Condens. Matter 9, 9113 (1997).

[27] J. Champion et al., Phys. Rev. B 64, 140407 (2001).

[28] J.R. Stewart et al., J. Phys. Condens. Matter 16, L321 (2004).

[29] M.R. McHenry, B. G. Silbernagel, and J.H. Wernick, Phys. Rev. B 5, 2958 (1972).

[30] L. R. Walker and R. Walstedt, Phys. Rev. B 22, 3816 (1980).

[31] Y. J. Uemura et al., Phys. Rev. B 31, 546 (1985).

[32] J. S. Gardner et al., J. Phys. Condens. Matter 16, S643 (2004).

[33] P. Dalmas de Réotier and A. Yaouanc, Phys. Rev. B 52, 9155 (1995).

[34] A. Yaouanc et al., Phys. Rev. B 53, 350 (1996).

[35] It is possible to get $\lambda_{Z}$ temperature independent using the conventional dispersion relation for antiferromagnets $\epsilon=$ $c_{\mathrm{AF}} q$ with $c_{\mathrm{AF}} \propto T^{5 / 6}$. However, this leads to a specific heat proportional to $T^{1 / 2}$, in disagreement with experiment. The proportionality factor leads also to much higher values for the low temperature specific heat than actually measured.

[36] P.W. Anderson, Basic Notions of Condensed Matter Physics (The Benjamin/Cummings Publishing Company, London, 1984).

[37] E. Rastelli, S. Regina, and A. Tassi, Phys. Rev. B 67, 094429 (2003).

[38] J. Lago et al., J. Phys. Condens. Matter 17, 979 (2005).

[39] J. Villain et al., J. Phys. (France) 41, 1263 (1980). 